\title{
CORRECTION
}

\section{Correction to: Hormonal modulation of ESR1 mutant metastasis}

Guowei Gu, Lin Tian (D), Sarah K. Herzog, Yassine Rechoum, Luca Gelsomino, Meng Gao, Lili Du, Jin-Ah Kim, Derek Dustin, Hin Ching Lo, Amanda R. Beyer, David G. Edwards, Thomas Gonzalez, Anna Tsimelzon, Helen J. Huang, Natalie M. Fernandez, Sandra L. Grimm, Susan G. Hilsenbeck, Dan Liu, Jun Xu, Alyssa Alaniz, Shunqiang Li, Gordon B. Mills (D), Filip Janku (D), Ralf Kittler, Xiang H. -F. Zhang (iD, Cristian Coarfa (D), Charles E. Foulds (D), W. Fraser Symmans (iD, Sebastiano Andò (D) and Suzanne A. W. Fuqua (D)

(c) The Author(s), under exclusive licence to Springer Nature Limited 2021

Oncogene (2022) 41:460; https://doi.org/10.1038/s41388-021-02104-w

Correction to: Oncogene (2021) 40:997-1011; https://doi.org/ 10.1038/s41388-020-01563-x, published online 15 December 2020
Grant number RP180712/CPRIT-MIRA was missing in the Funding information.

The original article has been corrected. 